\title{
Interrelation between Patient Satisfaction and Patient-Provider Communication in Diabetes Management
}

\author{
Ayse Basak Cinar ${ }^{1}$ and Lone Schou ${ }^{2}$ \\ ${ }^{1}$ Institute of Odontology, University of Copenhagen, Section 1, Norre Alle 20, 2200 Copenhagen, Denmark \\ ${ }^{2}$ Department of Global Oral Public Health, Institute of Odontology, University of Copenhagen, 2200 Copenhagen, Denmark \\ Correspondence should be addressed to Ayse Basak Cinar; aci@sund.ku.dk
}

Received 17 July 2014; Revised 29 October 2014; Accepted 19 November 2014; Published 28 December 2014

Academic Editor: Hugh Silk

Copyright (c) 2014 A. B. Cinar and L. Schou. This is an open access article distributed under the Creative Commons Attribution License, which permits unrestricted use, distribution, and reproduction in any medium, provided the original work is properly cited.

\begin{abstract}
The present study aims to assess how patient satisfaction with medical provider-patient communication can affect oral health, diabetes, and psychobehavioural measures among type 2 diabetes (T2DM) patients. It is part of a prospective intervention study among randomly selected T2DM patients, in Turkey. The data analyzed were Community Periodontal Need Index (CPI), HbAlc, patient satisfaction with communication, and psychobehavioural variables. Data was collected initially and at the end of the intervention. The participants were allocated to either health coaching (HC) or health education (HE). At baseline, there were no statistical differences between the $\mathrm{HC}$ and the HE groups on any of the measures $(P>0.05)$. Patients in both the HC and the HE groups had low satisfaction with communication. At postintervention, the increase in patient satisfaction with communication in the HC group was significantly higher than that in the HE group $(P=0.001)$. Principal component analysis revealed that patient satisfaction with communication shared the same cluster with clinical measures (CPI and HbAlc) and quality of life in the HC group. In conclusion, the present study showed, to our knowledge for the first time, that overall patient satisfaction with medical care provider-patient communication, empowered by HC approach, was interrelated with well-being of T2DM patients, in terms of psychobehavioural and clinical measures.
\end{abstract}

\section{Introduction}

The burden of diabetes is increasing globally, particularly in developing countries, and it is expected at least to have been doubled by 2030, where 694 million people are expected to suffer from diabetes [1]. The WHO projects that diabetes will be the 7 th leading cause of death in 2030 [2]. Type 2 diabetes (T2DM) comprises $90 \%$ of people with diabetes around the world [3] and is largely the result of poor lifestyles.

Patients with periodontal disease are more likely to have T2DM [4-6]; they have a higher risk for further complications such as cardiovascular problems $[7,8]$ and mortality at early age [9]. An effective prevention method for initiation/progression of periodontal diseases is daily toothbrushing [10], which is part of personal lifestyle. A good quality of patient-provider communication can positively affect the adoption of daily toothbrushing habits because patients value having a supportive and caring dentist and a dedicated dental team. Research has shown that the experience of having a dedicated, supportive, and caring dentist helped patients to take control of their own oral health [11]. However, in the literature, it is not clear how patient-provider communication can influence self-care practices, such as toothbrushing.

The majority of T2DM management is conducted by the patient [12] and diabetes is considered as one of the most psychologically and behaviourally demanding chronic diseases [13]. Many T2DM patients find themselves unable to follow recommended medical regimes and lifestyles (a healthy diet, regular physical exercise, twice daily toothbrushing, and no smoking), which makes them more prone to T2DM-related complications and poor oral health, leading to a poor quality 
of life $[12,14,15]$. Satisfaction with the patient-provider communication is one of the key elements of adherence to medical regimes [16] and diabetes outcomes [17].

Effective diabetes management requires effective communication between patient and provider, where medical professionals address psychosocial issues and are concerned about the expectations and needs of the patients. As professional diabetes care addresses multidisciplinary team approach (physician, dietician, psychologist, etc.), patients' satisfaction with communication refers to overall satisfaction with the multidisciplinary team of diabetes care. That may have a significant impact on their adherence to medical regimes and adoption of healthy lifestyles. However, the interrelation between patient overall satisfaction with a multidisciplinary team and diabetes management has been a neglected issue. The present study aims to assess how overall patient satisfaction with medical provider- (dentist- and physician-) patient communication can affect oral health, diabetes, and psychobehavioural measures among T2DM patients.

\section{Material and Methods}

The present study is part of a prospective intervention study among T2DM patients $(n=186)$, randomly selected from the outpatient clinics of two hospitals in Istanbul, Turkey. The power and sample size has been explained previously [1820]. Eligibility criteria were (1) confirmed type II diabetes, (2) 30-65-year-olds with at least 4 functional teeth, and (3) no psychological treatment and hospitalization.

Ethical approval and written permission to conduct the study were granted by the Ministry of Health. Information regarding $\mathrm{HbAlc}$ was taken from the latest medical records at the hospital, while the patients were invited for periodontal clinical examination. Of the patients participating, $96 \%$ (baseline visit, $n=179$; final visit, $n=178$ ) attended the clinical examinations. Of 186 participants, the dropout was 7 patients (4\%) and the corresponding figure for the participants who did not regularly participate in all sessions was $24(13 \%)$.

Back translations to and from Turkish were performed for the health behavior questionnaires by two native speakers to ensure comparability with the original forms in English.

The data in the present study were extracted from the self-assessed questionnaires, clinical measurements, and the medical records taken at baseline and at the end of follow-up. At the baseline visit, participants provided informed consent and filled out questionnaires (including demographic background and psychosocial and behavioural variables). The last current medical reports (glycated haemoglobin expressed as the percentage of haemoglobin that is exposed to glucose (HbAlc), HDL, LDL, and triglyceride) were drawn from the hospital. Subsequently all participants were invited for baseline oral examination which was conducted by two calibrated examiners. Examiners were blind to group assignments.

Following the baseline oral examinations, patients were randomly assigned to either the health coaching (HC) or the health education (HE) groups by means of a block table of random numbers (block randomization by physician).
The procedure and randomization were explained in detail previously [18-20]. Following the oral examination, patients entered $\mathrm{HC}$ or $\mathrm{HE}$ groups as explained above. The study included two phases (10-month initiation and maintenance and a 6-month follow-up). During the 10-month initiation and maintenance, all participants in both groups were invited for free periodontal cleaning and three seminars about oral health and diabetes management. Periodontal cleaning was offered free of charge and it was performed by a dentist (BEA) at a dental polyclinic; there were no oral health care facilities available at the hospitals. In connection with the periodontal cleaning to all participants, standard oral health education was given. All patients were called between one and three times for an appointment. The cleaning included the removal of soft and calcified deposits by an ultrasonic device. At the end of the 6-month follow-up phase, the same outcome measures were collected.

2.1. Health Coaching Group. One professional health coach (ABC) provided $\mathrm{HC}$ intervention for the participants. ABC had an internationally accredited coaching training. Participants randomized to the $\mathrm{HC}$ had a face-to-face session with the coach within 2 weeks of the baseline visit.

$\mathrm{HC}$, described earlier in detail [18], is focused on empowerment of patients for daily health-related practices, compliance to diabetes and oral health-related self-care regimes, building up health-related capacity skills, self-monitoring, and taking responsibility of health and quality of life. Besides, it increasingly targets the awareness of patients for risk factors and possible complications considering diabetes and oral diseases. The primary method is that patients set up the goal and an action plan, focusing on improvement of lifestyle and clinical measures, under the supervision of the coach. Each coaching session is used for subsequent monitoring of patients' progress towards the achievement of the target goal. Preset time frame for face-to-face coaching sessions is 20-60 minutes, determined by needs, expectations, hindrances, and progress of the patient.

HC was supported by educational and motivational brochures, designed for the project. Brochures in the format of written text were given to each patient. Two copies of the brochures were given to the leader and the head nurses of the polyclinics of the hospitals for information and any possible comments. Later on, the brochures were used at "Diabetes School Seminars" by the hospitals.

2.2. The Health Education (HE) Group. Participants randomized to the HE group received standard lifestyle advice referring to oral health care practices, diet, and physical exercise. One dentist provided HE intervention. The HE group patients were supported by the same educational brochures as the HC group.

All participants in both the $\mathrm{HC}$ and $\mathrm{HE}$ groups were supported by 8 text messages at the initiation phases and on special occasions (Ramadan fests, New Year Eve). They also received health promotion samples (toothbrush, toothpaste, sweeteners, and Pilates exercise balls). 
2.3. Outcome Variables. Oral examination, in particular periodontal examination, was performed by two calibrated clinicians [18-20]. The WHO periodontal probe with a 0.5 $\mathrm{mm}$ ball tip was used to determine Community Periodontal Need Index (CPI) by measuring the pocket depth and to detect subgingival calculus and bleeding response. The index teeth at six sextants $(17,16,11,26,27,47,46,31,36$, and 37) were probed and the highest score for each of the six sextants was recorded, in line with WHO recommendations for measurement of CPI $[21,22]$. If no index teeth or tooth was present in a sextant qualifying for examination, at least two nonindex remaining teeth in that sextant were examined and the highest score was recorded for each sextant.

Diabetes management was measured in terms of HbAlc. The respective variables were taken from the last health records, provided by either the hospital or the participants.

Patient satisfaction with the medical provider-patient communication was measured by a scale composed of 4 items ranging on a 5 -point Likert Scale (" $0=$ very unsatisfied" to " $4=$ very satisfied"). The question "How satisfied are you with your ... during the last 4 months?" was used to assess the patient satisfaction on the (1) attitude of the physician in general, (2) information/counselling about diabetes management provided by the physician, (3) attitude of the dentist in general, and (4) information/counselling about the role of diabetes in oral health management provided by the dentist. The Cronbach correlation coefficient measures $(\alpha=$ 0.701 ) were good. Further, the split-half internal consistency, applied to test the reliability of the scale, was 0.68 (EqualLength Spearman-Brown) with the correlation between the two halves being $r=0.81$.

Questions about self-reported physical activity were taken from an earlier study [23]. Participants answered a multiple choice question like "Please tick the activity that fits you best." There were four answer possibilities: "(1) read, watch TV or other things in a sitting position, (2) walking, active house work at least four hours per week, (3) jogging, running and other kind of running exercises or hard work in a garden 2-3 hours per week, (4) tough training, competition, sport more than once a week." Responses were reclassified into three categories by taking the last two categories as one "physically high active" as the responses to each of these categories were not so high. For further analysis, it was dichotomized as "physically inactive" and "physically active."

2.4. Psychobehavioural Measures. Self-reported toothbrushing frequency was taken from an earlier study [14] asking the following: "How often do you brush teeth?" Toothbrushing frequency was recorded on a 5-point Likert Scale ("never $=0$, once a week/less $=1,2-5$ times/week $=2$, once daily $=3$, twice/more daily $=4 "$ "). For further analysis, it was dichotomized as "irregular: brushing less than once a day" and "regular: brushing at least once a day".

The toothbrushing self-efficacy (TBSE) scale [14, 19, 24] was used to assess individual's belief in his/her competency to brush his/her teeth daily across different challenging situations by the question "How sure are you that you can brush your teeth...." TBSE scale consisted of eight items on a five-point Likert Scale (0: "not sure at all" to 5: "absolutely sure"). (For the design and validity-reliability measures of the scale, see $[14,19,24])$.

The quality of life regarding daily diabetes management was modified version of the WHOQOL-Bref $[25,26]$. It referred to the physical and psychological domains (Table 1). It included 6 items: 3 physical and 3 psychological well-beingrelated item (responses on a 5-point Likert Scale; 0: "not at all" to 4: "very extreme amount" for the items 1 and 2 and reverse coding for the items 3-5, and 4: "never" to 0: "always" for the item 6). The validity and the reliability of the scale were tested earlier [25].

2.5. Analysis. Statistical analyses were performed using SPSS v.17 (Chicago, Illinois). For assessment of correlation and baseline similarities/differences between the $\mathrm{HC}$ and the $\mathrm{HE}$ groups, respectively, Spearman rank correlation, independent sample $t$-test and cross-tabulation, and chi square analysis were used. Statistical significance was set at 0.05 for each test.

Factor analysis can be used to hypothesize an underlying construct by the principal component analysis (PCA) approach; thus, it is used to find a few combinations of variables, called components or clusters, which adequately explain the overall observed variation, thereby reducing the complexity of the data, as described in an earlier study [24]. In the present study, factor analysis was applied to the variables by using PCA and Varimax rotation to analyze not the associations but the interrelationships (connection by sharing the common background factors) and common underlying dimensions among patient satisfaction with patient-provider communication, periodontal health, and HbAlc levels and psychobehavioural variables (self-efficacy, quality of life, physical activity, and toothbrushing). These variables were classified into discriminative clusters (latent variables) based on factorial loadings, ranging from highest to lowest values. Loadings below 0.25 were extracted for ease of communication. The clusters are named based on the variable with highest loading. Factors were extracted according to meeting the Kaiser criterion of eigenvalue greater than 1 .

\section{Results}

At baseline, there were no statistical differences between the HC and the HE groups on the oral health- and diabetes related measures $(P>0.05)$ (Table 2$)$. Patients in both the $\mathrm{HC}$ and the HE groups had low satisfaction with medical provider-patient communication (mean: $6.3 \pm 3.7$ versus $7.3 \pm$ $3.9 P>0.05)$.

At postintervention, the increase at satisfaction with the communication in the HC group (mean change $=6.3$ ) was significantly higher than that in the HE group (mean change $=$ $3.1),(P=0.001)$ (Table 2). The HC group patients, who were highly satisfied with medical provider-patient communication, were more likely to be physically active (94\%) than those who were less satisfied $(67 \%)(P=0.001)$. Such a significant association was not observed in the HE group. In the HC group, those who were physically active were more likely to brush their teeth regularly (92\%) compared to those who were 
TABLE 1: The quality of life regarding daily diabetes management (modified WHOQOL-Bref).

\begin{tabular}{|c|c|c|c|c|c|}
\hline \multicolumn{6}{|c|}{ To which extent or how much have you experienced certain things in the last 4 weeks? } \\
\hline & Not at all & Little & $\begin{array}{l}\text { A moderate } \\
\text { amount }\end{array}$ & Very much & $\begin{array}{l}\text { An extreme } \\
\text { amount }\end{array}$ \\
\hline \multicolumn{6}{|l|}{$\begin{array}{l}\text { To what extent do you feel } \\
\text { that physical pain prevents } \\
\text { you from doing what you } \\
\text { need to do? }\end{array}$} \\
\hline \multicolumn{6}{|l|}{$\begin{array}{l}\text { How much do you need } \\
\text { any medical treatment to } \\
\text { function in your daily life? }\end{array}$} \\
\hline \multicolumn{6}{|l|}{$\begin{array}{l}\text { Do you have enough } \\
\text { energy for everyday life? }\end{array}$} \\
\hline \multicolumn{6}{|l|}{$\begin{array}{l}\text { How much do you enjoy } \\
\text { life? }\end{array}$} \\
\hline \multicolumn{6}{|l|}{$\begin{array}{l}\text { To what extent do you feel } \\
\text { that your life is meaningful? }\end{array}$} \\
\hline $\begin{array}{l}\text { How often do you feel } \\
\text { hopeless, depressed, or } \\
\text { anxious? }\end{array}$ & & & & & \\
\hline
\end{tabular}

physically inactive $(64 \%)(P=0.001)$. Such a significant association was not observed in the HE group $(P>0.05)$.

Principal component analysis revealed that patient satisfaction with the medical provider-patient communication shared the same cluster with oral health- and diabetes related measures and quality of life among the HC group (Table 3). In the HE group, patient satisfaction with the medical provider-patient communication was interrelated with CPI and HbAlc. Patient satisfaction with providerpatient communication in the HE group was correlated by improvement at self-efficacy $\left(r_{s}=0.295\right)$ and quality of life $\left(r_{s}=0.366\right)(P<0.05)$.

\section{Discussion}

We showed that satisfaction of T2DM patients with providerpatient communication is interrelated with health outcomes, determined by HbAlc and CPI in both the HC group and HE group. These findings may add to the growing body of evidence linking patient satisfaction with diabetes related outcomes $[17,27,28]$. The dental literature, scarce in number, suggests that dentist-patient communication directly affects patient satisfaction with the dental treatment $[29,30]$ or oral health services [31]. However, it is not known how that communication may affect oral health and/or behaviour. The present study shows how overall satisfaction with patientprovider communication is interrelated with both oral health and diabetes.

HC focuses on self-empowerment and it is a patientfocused approach for transformation and maintenance of healthy lifestyles and to improve quality of life. HC helps individuals to transform their cognitive and emotional functioning to adopt positive health behaviours, by setting up personal goals and specific action plans, supported by improvement of self-efficacy. Self-efficacy focuses on one's confidence to perform a given behaviour [32]. Several studies have documented associations between self-efficacy and diabetes self-care [33-35]. Increased self-efficacy and thereby better diabetes management are related with engagement in shared decision making for medical care/treatment and better quality of communication between patient and provider [36]. In the present study, in the $\mathrm{HC}$ group, satisfaction with patient-provider communication, oral health and $\mathrm{HbAlc}$, self-efficacy, and quality of life formed a single cluster. That may reveal patients whose self-efficacy improved through coaching intervention took decisions and acted on to improve their oral health and diabetes and thereby improved their quality of life. Increased awareness and self-confidence in their capabilities to improve their quality of life may lead these patients to engage actively in medical consultations/visits. Moreover, patients who take a more active role often are more satisfied with care and more committed to treatment plans, have a better understanding of treatment options, and experience greater improvement in health than do more passive patients [37]. That may also be an explanatory factor for why the patients in the HE group did not have any improvements in the clinical measures. Those patients had less improvement in the self-efficacy; thereby, they most probably did not take an active role in decision making during consultations which may lead to less satisfaction with the communication.

T2DM patients who discuss their treatment goals and management strategies with their physicians tend to have better clinical outcomes than those who do not [38, 39]. The individually tailored and goal oriented programs, particularly based on improving self-efficacy, have been more efficacious in improving periodontal health compared to the education group [40-43]. Therefore, goal setting and empowerment by self-efficacy can be defined as the key core for better health outcomes. In the present study, HC focused on selfempowerment and helped patients to set up health-related 


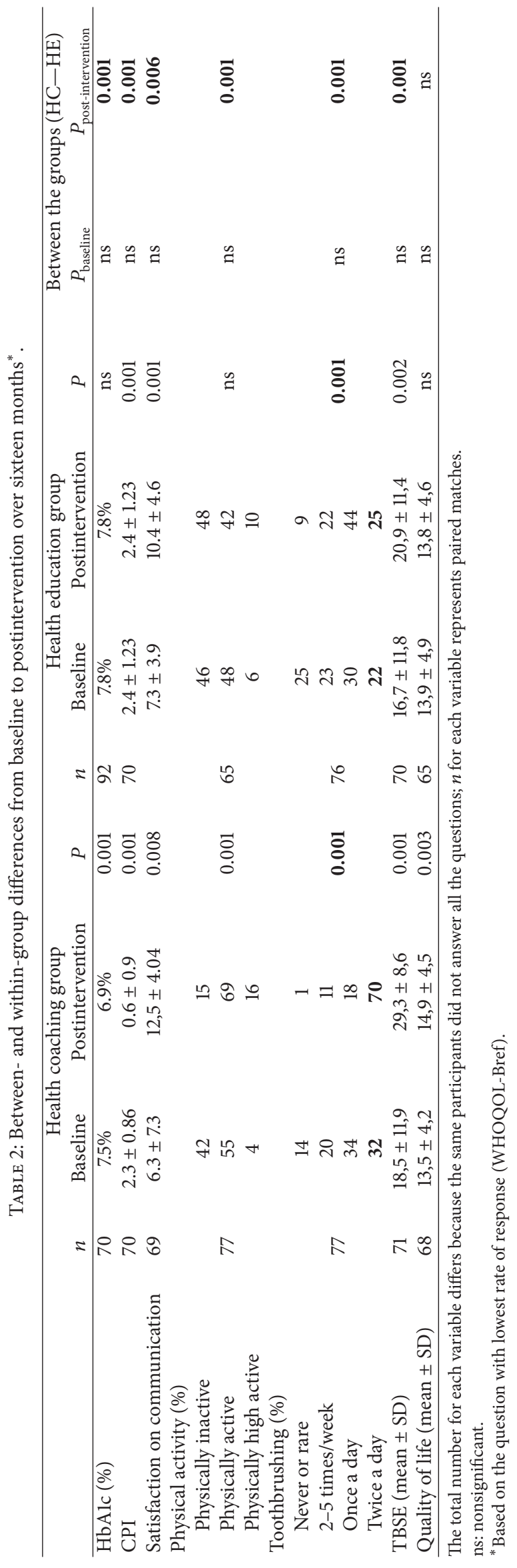


TABle 3: Principal component analysis for assessing the clusters of patient satisfaction with provider-patient communication among patients with type 2 diabetes in the (a) health coaching group and in the (b) health education group.

(a) Health coaching group

\begin{tabular}{lc}
\hline & Quality of life \\
\hline Satisfaction with provider-patient & 0,693 \\
communication & $-0,678$ \\
CPI (periodontal treatment need) & $-0,610$ \\
HbAlc & 0,449 \\
Toothbrushing self-efficacy & 0,760 \\
Quality of life
\end{tabular}

The clusters in the study group, in total, accounted for $41.8 \%$ of the total variance. The cluster is named based on variable with the highest loading.

(b) Health education group

\begin{tabular}{lcc}
\hline & Oral health & Quality of life \\
\hline $\begin{array}{l}\text { Satisfaction with } \\
\text { provider-patient } \\
\text { communication }\end{array}$ & 0,555 & 0,361 \\
CPI (periodontal treatment & $-0,765$ & $*$ \\
need) & $-0,717$ & 0,527 \\
HbAlc & $*$ & 0,544 \\
Toothbrushing self-efficacy & $*$ & 0,840 \\
Quality of life &
\end{tabular}

The clusters in the study group, in total, accounted for $56.5 \%$ of the total variance [composed of component 1 (oral health): $30.7 \%$ and component 2 (quality of life): $25.8 \%]$.

${ }^{*}$ Loadings below 0.25 extracted for ease of communication. The clusters are named based on the variable with the highest loading.

goals by using "Wheel of Health" which enabled patients to assess the interrelation between goals. Patients were followed up by coaching sessions where the motivation and empowerment to succeed the achievement were the key core. In the earlier study, we showed that patients who explored their self-management skills and competencies through HC sessions were more likely to attain healthy lifestyle; selfefficacy, physical activity, and toothbrushing shared one cluster. In the present study, satisfaction with the providerpatient communication was positively correlated with being physically active and regular toothbrushing in the HC group. Another way of describing, patients who were taking responsibility of their life by engaging in healthy lifestyles were more likely to be satisfied with their communication with their medical care providers. An explanation may be that HC, based on "learn-act-grow," motivated patient to learn new information and to use his/her resources and then to act on behavioural change by unlocking his/her initial potential to grow continuously. This most probably led the patients in HC group to be actively involved in medical consultations, thereby learning and growing by taking the answers based on their own needs and expectations that led to an increased satisfaction. However, that requires further studies to explore the underlying pathways. There seems to be bilateral relationship between attaining healthy lifestyles and satisfaction with provider-patient communication. Therefore, it is important that medical professionals support patient motivation and help to empower engagement in healthy lifestyles, in particular considering the paradigm stating that patient satisfaction is a proxy measure for health $[44,45]$.

A limitation of the present study is the small sample size. Due to a number of organisational challenges, personnel, training, funding, time, and so forth, it was not possible to increase the number of participants. However, the original sample size is within the range of sample sizes of the studies in the field. Even though the sample is small and it is not representative of the general population of T2DM patients in Turkey, the study could be a model for further studies. Another limitation is that we did not measure the content of medical consultations to assess the differences between the $\mathrm{HC}$ and the HE groups; however, this was not in the scope of the intervention. Further studies need to explore the content and change in the content of medical/dental consultations. However, the study is, to our knowledge, the first of its kind which analyses the interrelation between patient's overall satisfaction with medical provider-patient communication on oral health and diabetes and psychobehavioural outcomes. The strengths of the study are, as discussed earlier by Cinar et al. [18], that (1) it has a comparison group (HC versus $\mathrm{HE}),(2)$ it has a relatively long period of intervention (16 months including a follow-up), (3) it is structured and uses internationally accredited content of HC, (4) it uses a validityreliability tested self-efficacy measurement instrument, and (5) the health goals were set up by patients, thereby prioritizing the patients' own need and expectations. Furthermore, all HbAlc measures were taken from the records of the hospitals, so there was not any bias from self-reports.

\section{Conclusion}

The present study, for the first time to our knowledge, assessed that overall patient satisfaction with medical provider- (dentist- and physician-) patient communication was interrelated with oral health, diabetes, and psychobehavioural measures among T2DM patients, varying in different pathways in the $\mathrm{HC}$ and the HE groups. Our findings on the differentiation at pathways (clusterization) between HC and HE group can reveal that there is a threshold for patient satisfaction with medical provider-patient communication; above this threshold, personal measures such as self-efficacy and quality of life can be positively affected. HC in the present study significantly improved patient satisfaction with medical provider communication which was interrelated with health as a whole, in terms of clinical and psychobehavioural measures. HE improved satisfaction on communication with medical provider to a certain extent and that was interrelated only with clinical measures. The impact of HE does not seem to go deep or in another way of saying it cannot unlock the initial potential of the patient (self-competency); so the holistic interaction and improvement in clinical and personal measures seem to be lacking. There is a need for further studies to assess the impact of patient empowerment based $\mathrm{HC}$ on medical provider-patient communication, in 
particular on the content and interaction patterns between patient and the medical provider.

The results will hopefully encourage stakeholders and politicians to gain new insights for setting and supporting patient focused health promoting programmes like $\mathrm{HC}$ in primary health care settings especially for T2DM patients. This may facilitate dissemination, in order to improve both clinical and personal health outcomes at an individual and public health level, thereby helping to reduce the burden of T2DM. The HC methodology integrated programmes can significantly take part in reversing this epidemic.

\section{Conflict of Interests}

The authors declare that there is no conflict of interests regarding the publication of this paper.

\section{Acknowledgments}

The authors express their deepest thanks to Professor Nazif Bagriacik (Head, Turkish Diabetes Association) and Associate Professor Mehmet Sargin and Head Diabetes Nurse Sengul Isik (Diabetes Unit, S. B. Kartal Research and Education Hospital) for all their support and help during the research. They thank Professor Aytekin Oguz for his help on the preparation of the documents for the ethical permission. They also thank Professor I Oktay and periodontologist Duygu Ilhan for training for clinical oral examinations. They thank Christian Dinesen for coaching training of $\mathrm{AB}$ Cinar and for his support for the research. They also thank ZENDIUM for oral health care kits, SPLENDA (TR) for the promotional tools, ChiBall World Pty Ltd. for exercising chi-balls, and IVOCLAR Vivadent, Plandent, Denmark, for provisioning of CRT kits. Finally, many thanks are due to their patients for their participation and cooperation. The research is part of an international project that has two phases. The Turkish phase, which is presented here, is supported by FDI and the International Research Fund of University of Copenhagen. The second phase is conducted in Denmark. It is supported by BRIDGES and BRIDGES is an IDF program supported by an educational grant from Lilly Diabetes.

\section{References}

[1] WHO, Diabetes, Fact sheet no. 312, 2013, http://www.who.int/ mediacentre/factsheets/fs312/en/.

[2] World Health Organization, Global Status Report on Noncommunicable Diseases 2010, World Health Organization, Geneva, Switzerland, 2011.

[3] Definition, Diagnosis and Classification of Diabetes Mellitus and Its Complications. Part 1: Diagnosis and Classification of Diabetes Mellitus, WHO/NCD/NCS/99.2, World Health Organization, Geneva, Switzerland, 1999.

[4] H. Loe, "Periodontal disease: the sixth complication of diabetes mellitus," Diabetes Care, vol. 16, no. 1, pp. 329-334, 1993.

[5] G. E. Sandberg, H. E. Sundberg, C. A. Fjellstrom, and K. F. Wikblad, "Type 2 diabetes and oral health: a comparison between diabetic and non- diabetic subjects," Diabetes Research and Clinical Practice, vol. 50, no. 1, pp. 27-34, 2000.
[6] P. Güneri, F. Ünlü, B. Yeșilbek et al., "Vascular endothelial growth factor in gingival tissues and crevicular fluids of diabetic and healthy periodontal patients," Journal of Periodontology, vol. 75, no. 1, pp. 91-97, 2004.

[7] R. J. Genco, I. Glurich, V. Haraszthy, J. Zambon, and E. DeNardin, "Overview of risk factors for periodontal disease and implications for diabetes and cardiovascular disease," Compendium of Continuing Education in Dentistry, vol. 22, no. 2, pp. $21-23,2001$.

[8] M. Marjanovic and K. Buhlin, "Periodontal and systemic diseases among Swedish dental school patients-a retrospective register study," Oral Health \& Preventive Dentistry, vol. 11, no. 1, pp. 49-55, 2013.

[9] K. Avlund, K. Schultz-Larsen, U. Krustrup, N. Christiansen, and P. Holm-Pedersen, "Effect of inflammation in the periodontium in early old age on mortality at 21-year follow-up," Journal of the American Geriatrics Society, vol. 57, no. 7, pp. 1206-1212, 2009.

[10] H. Löe, "Ural hygiene in the prevention of caries and periodontal disease," International Dental Journal, vol. 50, no. 3, pp. 129$139,2000$.

[11] A. Sbaraini, S. M. Carter, R. W. Evans, and A. Blinkhorn, "Experiences of dental care: what do patients value?" BMC Health Services Research, vol. 12, no. 1, article 177, 2012.

[12] L. Minet, S. Møller, W. Vach, L. Wagner, and J. E. Henriksen, "Mediating the effect of self-care management intervention in type 2 diabetes: a meta-analysis of 47 randomised controlled trials," Patient Education and Counseling, vol. 80, no. 1, pp. 2941, 2010.

[13] D. J. Cox and L. Gonder-Frederick, "Major developments in behavioral diabetes research," Journal of Consulting and Clinical Psychology, vol. 60, no. 4, pp. 628-638, 1992.

[14] A. B. Cinar, Preadolescents and their mothers as oral healthpromoting actors: non-biologic determinants of oral health among Turkish and Finnish preadolescents [Ph.D. thesis], University of Helsinki, Helsinki, Finland, 2008.

[15] WHO, Adherence to Long-Term Therapies: Evidence for Action, 2003.

[16] P. S. Ciechanowski, W. J. Katon, J. E. Russo, and E. A. Walker, "The patient-provider relationship: attachment theory and adherence to treatment in diabetes," American Journal of Psychiatry, vol. 158, no. 1, pp. 29-35, 2001.

[17] M. H. Alazri and R. D. Neal, "The association between satisfaction with services provided in primary care and outcomes in Type 2 diabetes mellitus," Diabetic Medicine, vol. 20, no. 6, pp. 486-490, 2003.

[18] A. B. Cinar, I. Oktay, and L. Schou, "Smile healthy to your diabetes': health coaching-based intervention for oral health and diabetes management," Clinical Oral Investigations, vol. 18, no. 7, pp. 1793-1801, 2014

[19] A. B. Cinar and L. Schou, "The role of self-efficacy in health coaching and health education for patients with type 2 diabetes," International Dental Journal, vol. 64, no. 3, pp. 155-163, 2014.

[20] A. B. Cinar and L. Schou, "Health promotion for patients with diabetes: health coaching or formal health education?" International Dental Journal, vol. 64, no. 1, pp. 20-28, 2014.

[21] WHO, Oral Health Surveys: Basic Methods, WHO, Geneva, Switzerland, 1997.

[22] T. W. Cutress, J. Ainamo, and J. Sardo-Infirri, "The community periodontal index of treatment needs (CPITN) procedure for population groups and individuals," International Dental Journal, vol. 37, no. 4, pp. 222-233, 1987. 
[23] L. B. Christensen, P. E. Petersen, and B. Hede, "Oral health in children in Denmark under different public dental health care schemes," Community Dental Health, vol. 27, no. 2, pp. 94-101, 2010.

[24] C. A. Basak, K. Nilufer, and H. Murtomaa, "Self-efficacy perspective on oral health among Turkish pre-adolescents," Oral Health \& Preventive Dentistry, vol. 3, no. 4, pp. 209-215, 2005.

[25] A. B. Cinar, I. Oktay, and L. Schou, "Relationship between oral health, diabetes management and sleep apnea," Clinical Oral Investigations, vol. 17, no. 3, pp. 967-974, 2013.

[26] WHO, WHO Quality of Life-BREF (WHOQOL-BREF), 2004, http://www.who.int/substance_abuse/research_tools/whoqolbref/en/index.html.

[27] J. Gao, J. Wang, P. Zheng et al., "Effects of self-care, selfefficacy, social support on glycemic control in adults with type 2 diabetes," BMC Family Practice, vol. 14, article 66, 2013.

[28] W. K. Redekop, M. A. Koopmanschap, R. P. Stolk, G. E. H. M. Rutten, B. H. R. Wolffenbuttel, and L. W. Niessen, "Healthrelated quality of life and treatment satisfaction in Dutch patients with type 2 diabetes," Diabetes Care, vol. 25, no. 3, pp. 458-463, 2002.

[29] P. Gürdal, H. Çankaya, E. Önem, S. Dinçer, and T. Yilmaz, "Factors of patient satisfaction/dissatisfaction in a dental faculty outpatient clinic in Turkey," Community Dentistry and Oral Epidemiology, vol. 28, no. 6, pp. 461-469, 2000.

[30] K. Sondell, B. Söderfeldt, and S. Palmqvist, "Dentist-patient communication and patient satisfaction in prosthetic dentistry," International Journal of Prosthodontics, vol. 15, no. 1, pp. 28-37, 2002.

[31] I. Okullo, A. N. Åstrom, and O. Haugejorden, "Influence of perceived provider performance on satisfaction with oral health care among adolescents," Community Dentistry and Oral Epidemiology, vol. 32, no. 6, pp. 447-455, 2004.

[32] A. Bandura, Social Foundations of Thought and Action: A Social Cognitive Theory, Prentice-Hall, New York, NY, USA, 1986.

[33] O. A. Al-Khawaldeh, M. A. Al-Hassan, and E. S. Froelicher, "Self-efficacy, self-management, and glycemic control in adults with type 2 diabetes mellitus," Journal of Diabetes and Its Complications, vol. 26, no. 1, pp. 10-16, 2012.

[34] V. D. Sousa, J. A. Zauszniewski, C. M. Musil, P. J. Price Lea, and S. A. Davis, "Relationships among self-care agency, self-efficacy, self-care, and glycemic control," Research and theory for nursing practice, vol. 19, no. 3, pp. 217-230, 2005.

[35] C. H. Johnston-Brooks, M. A. Lewis, and S. Garg, "Self-efficacy impacts self-care and HbAlc in young adults with Type I diabetes," Psychosomatic Medicine, vol. 64, no. 1, pp. 43-51, 2002.

[36] M. Heisler, R. R. Bouknight, R. A. Hayward, D. M. Smith, and E. A. Kerr, "The relative importance of physician communication, participatory decision making, and patient understanding in diabetes self-management," Journal of General Internal Medicine, vol. 17, no. 4, pp. 243-252, 2002.

[37] R. L. Street Jr., H. S. Gordon, M. M. Ward, E. Krupat, and R. L. Kravitz, "Patient participation in medical consultations: why some patients are more involved than others," Medical Care, vol. 43, no. 10, pp. 960-969, 2005.

[38] N. D. F. Olivarius, H. Beck-Nielsen, A. H. Andreasen, M. Hørder, and P. A. Pedersen, "Randomised controlled trial of structured personal care of type 2 diabetes mellitus," British Medical Journal, vol. 323, no. 7319, pp. 970-975, 2001.

[39] A. D. Naik, N. Palmer, N. J. Petersen et al., "Comparative effectiveness of goal setting in diabetes mellitus group clinics: randomized clinical trial," Archives of Internal Medicine, vol. 171, no. 5, pp. 453-459, 2011.

[40] N. Kakudate, M. Morita, M. Sugai, and M. Kawanami, "Systematic cognitive behavioral approach for oral hygiene instruction: a short-term study," Patient Education and Counseling, vol. 74, no. 2, pp. 191-196, 2009.

[41] F. Almomani, K. Williams, D. Catley, and C. Brown, "Effects of an oral health promotion program in people with mental illness," Journal of Dental Research, vol. 88, no. 7, pp. 648-652, 2009.

[42] P. Philippot, N. Lenoir, W. D’Hoore, and P. Bercy, "Improving patients' compliance with the treatment of periodontitis: a controlled study of behavioural intervention," Journal of Clinical Periodontology, vol. 32, no. 6, pp. 653-658, 2005.

[43] B. Jönsson, K. Ohrn, N. Oscarson, and P. Lindberg, "An individually tailored treatment programme for improved oral hygiene: introduction of a new course of action in health education for patients with periodontitis," International Journal of Dental Hygiene, vol. 7, no. 3, pp. 166-175, 2009.

[44] B. Williams, "Patient satisfaction: a valid concept?" Social Science and Medicine, vol. 38, no. 4, pp. 509-516, 1994.

[45] B. Prakash, "Patient satisfaction," Journal of Cutaneous and Aesthetic Surgery, vol. 3, no. 3, pp. 151-155, 2010. 


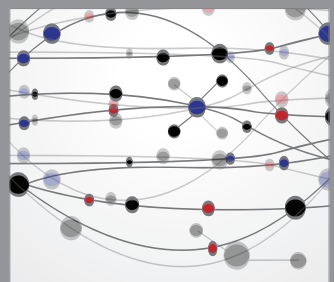

The Scientific World Journal
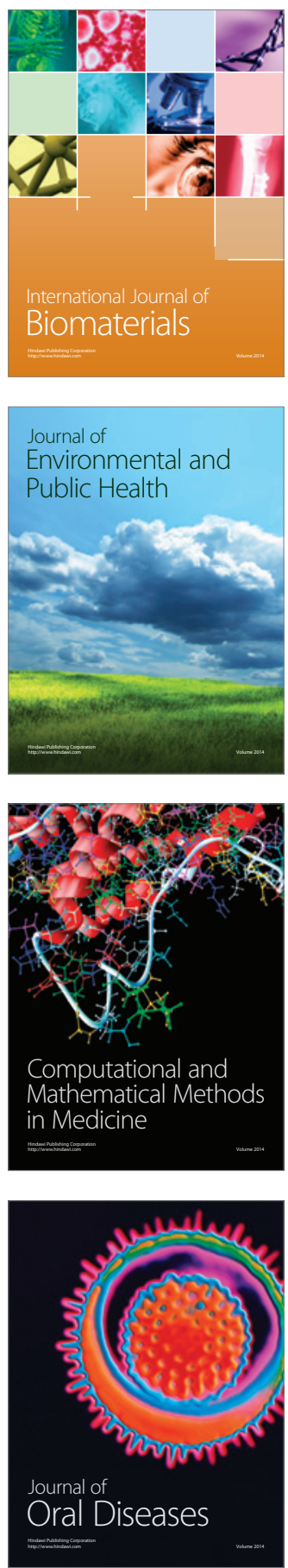
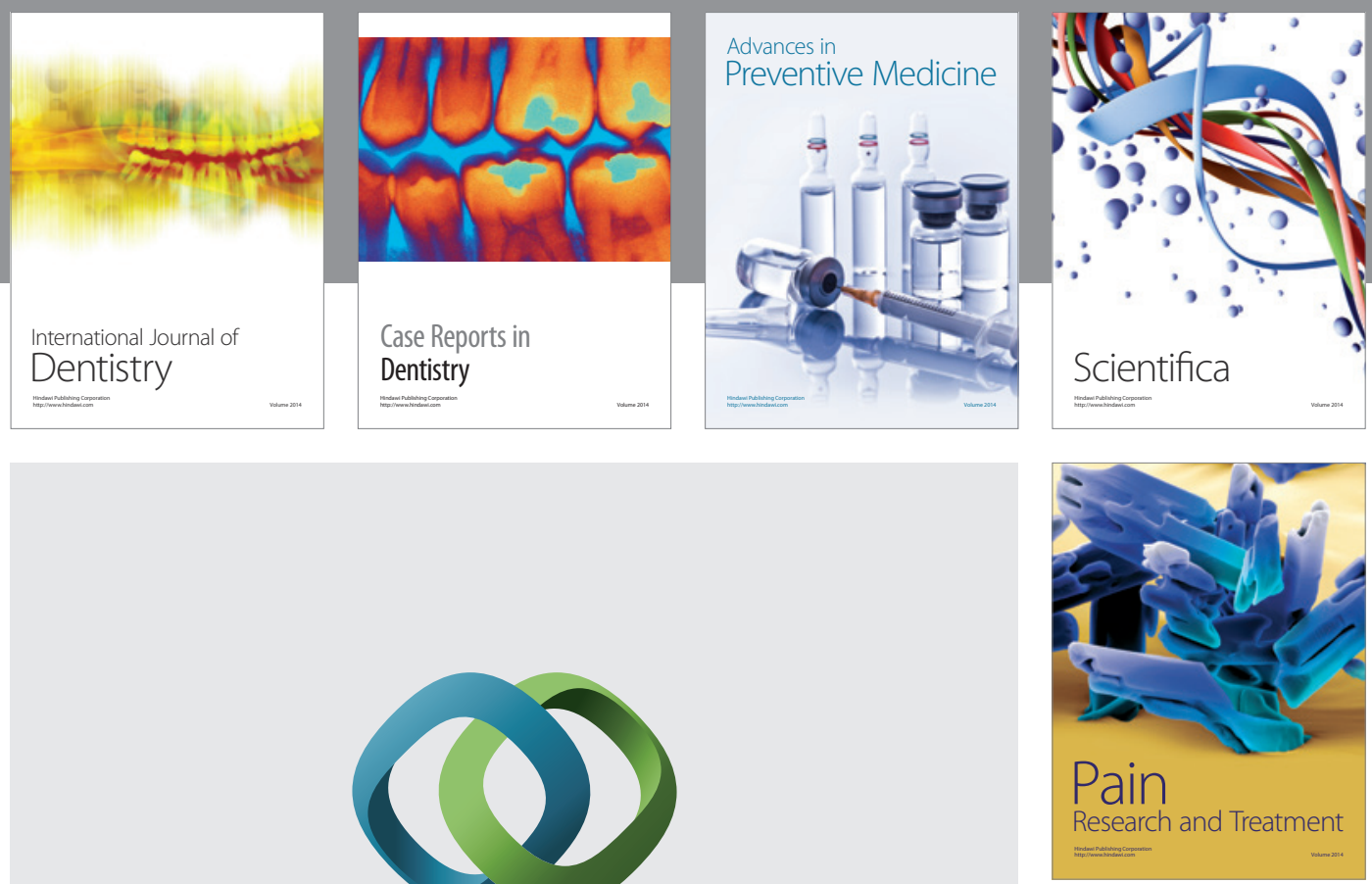

\section{Hindawi}

Submit your manuscripts at

http://www.hindawi.com
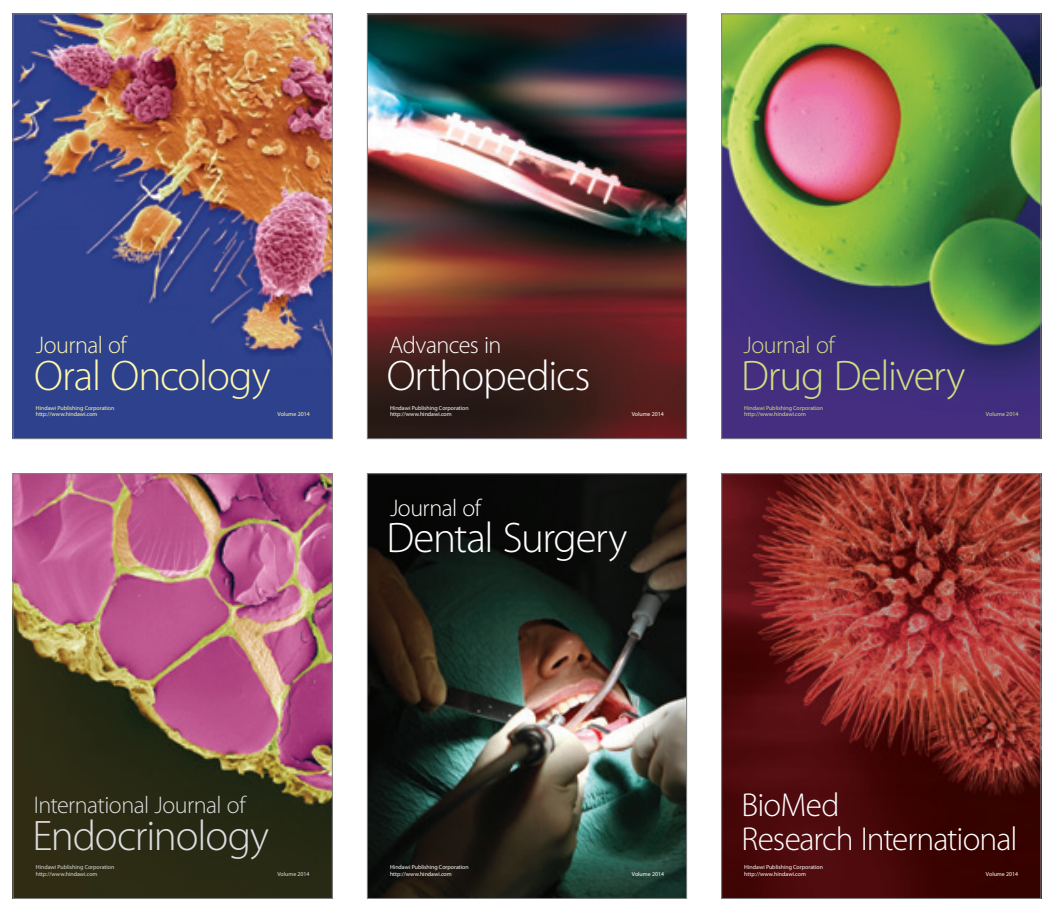

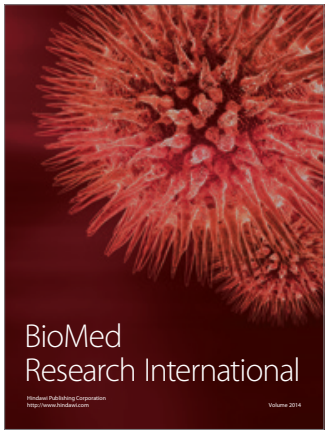

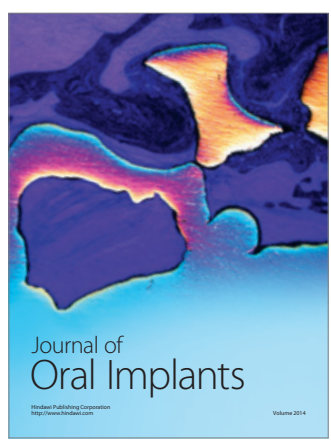
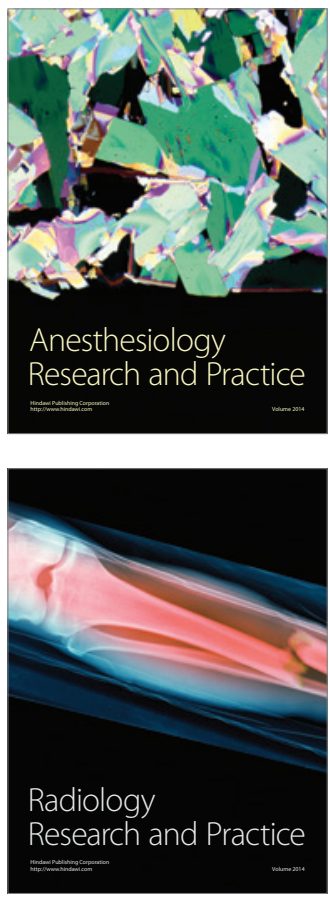